\title{
Evaluation of Respiratory Muscle Activity by Means of Concentric Ring Electrodes
}

\author{
Luis Estrada-Petrocelli, Senior Member, IEEE, Abel Torres, Senior Member, IEEE, Leonardo \\ Sarlabous, Magda Ràfols-de-Urquía, Yiyao Ye-Lin, Gema Prats-Boluda, Member, IEEE, \\ Raimon Jané, Senior Member, IEEE and Javier Garcia-Casado, Member, IEEE
}

\begin{abstract}
Surface electromyography (sEMG) can be used for the evaluation of respiratory muscle activity. Recording SEMG involves the use of surface electrodes in a bipolar configuration. However, electrocardiographic (ECG) interference and electrode orientation represent considerable drawbacks to bipolar acquisition. As an alternative, concentric ring electrodes (CREs) can be used for SEMG acquisition and offer great potential for the evaluation of respiratory muscle activity due to their enhanced spatial resolution and simple placement protocol, which does not depend on muscle fiber orientation. The aim of this work was to analyze the performance of CREs during respiratory SEMG acquisitions. Respiratory muscle sEMG was applied to the diaphragm and sternocleidomastoid muscles using a bipolar and a CRE configuration. Thirty-two subjects underwent four inspiratory load spontaneous breathing tests which was repeated after interchanging the electrode positions. We calculated parameters such as (1) spectral power and (2) median frequency during inspiration, and power ratios of inspiratory SEMG without ECG in relation to (3) basal sEMG without ECG (Rins/noise), (4) basal sEMG
\end{abstract}

This work was supported in part by the CERCA Program/Generalitat de Catalunya, in part by the Secretaria d'Universitats i Recerca de la Generalitat de Catalunya under grant GRC 2017 SGR 01770 and in part by the spanish grants RTI2018-098472-B-I00, RTI2018-094449-AI00-AR (MCIU/AEI/FEDER, UE) and DPI2015-68397-R (MINECO/FEDER) and the Biomedical Research Networking Centre in Bioengineering, Biomaterials and Nanomedicine (CIBER-BBN, Instituto de Salud Carlos III/FEDER). The first author was supported by the IFARHU-SENACYT Scholarship Program from the Panama Government under Grant 270-2012-273. L. Estrada-Petrocelli, A. Torres, M. Ràfols-de-Urquía and R. Jané are with the Institut de Bioenginyeria de Catalunya (IBEC), the Barcelona Institute of Science and Technology (BIST). c/. Baldiri Reixac 4, 08028, Barcelona, Spain. (e-mails: \{atorres, lestrada, mrafols, rjane\}@ibecbarcelona.eu). L. Estrada-Petrocelli, A. Torres, L. Sarlabous and R. Jané are also with CIBER-BBN. A. Torres and R. Jané are also with the Universitat Politècnica de Catalunya (UPC)-BarcelonaTech, Barcelona, Spain. L. Estrada-Petrocelli is also with the Facultad de Ingeniería, Universidad Latina de Panamá, Panama, Panama. L. Sarlabous is with the Critical Care Center, Hospital Universitari Parc Taulí, Institut d'Investigació i Innovació Parc Taulí (I3PT), Universitat Autònoma de Barcelona, Sabadell, Spain (e-mail: Isarlabous@tauli.cat). Y. Ye-Lin, G. PratsBoluda and J. Garcia-Casado are with the Centro de Investigación e Innovación en Bioingeniería (Cl2B), Universitat Politècnica de València (UPV), Camino de la Vera s/n Ed. 8B, 46022 Valencia, Spain (emails: \{yiye, gprats, jgarciac\}@ci2b.upv.es). with ECG ( $\left.R_{\text {ins/cardio }}\right)$ and (5) expiratory sEMG without ECG (Rins/exp). Spectral power, $\mathbf{R}_{\text {ins/noise and }} \mathbf{R}_{\text {ins/cardio }}$ increased with the inspiratory load. Significantly higher values $(p<0.05)$ of $R_{\text {ins/cardio and significantly higher }}$ median frequencies were obtained for CREs. Rins/noise and

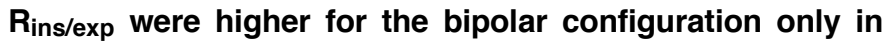
diaphragm SEMG recordings, whereas no significant differences were found in the sternocleidomastoid recordings. Our results suggest that the evaluation of respiratory muscle activity by means of $S E M G$ can benefit from the remarkably reduced influence of cardiac activity, the enhanced detection of the shift in frequency content and the axial isotropy of CREs which facilitates its placement.

Index Terms-Concentric ring electrodes, Laplacian potential, non-invasive respiratory monitoring, respiratory muscles, surface electromyography

\section{INTRODUCTION}

$R_{c}^{\circ}$ OUTINE measurements of respiratory function have contributed to the effective assessment of normal conditions in healthy subjects as well as to the effective diagnosis of patients with chronic respiratory diseases involving compromised respiratory airways and other structures of the lung. Spirometry is the pulmonary function test most commonly adopted in clinical practice, and it provides information on airway resistance and lung capacity based on measures of volume and airflow changes [1]. In addition, respiratory volume along with respiratory pressure measurements reflect the muscle respiratory load on the respiratory system, which in turn allows muscle respiratory function to be assessed [2]. A more objective measure of muscle respiratory function is provided by respiratory muscle electromyography (EMG).

Electromyography of the diaphragm muscle (EMGdi) records the electrical activity of the body's primary inspiratory muscle and provides a surrogate measure of the neural respiratory drive [3]. EMGdi can be obtained via a nasogastric tube with multi-pair electrodes positioned in the esophagus at the level of crural portion of the diaphragm. Measuring EMGdi, however, has several practical limitations including the fact that it requires advanced technical skills, which restrict its use to research environments [3]. Due to constraints in the use of invasive measures, researchers are exploring non- 
invasive alternatives based on the recording of respiratory muscle EMG using surface electrodes (sEMG) placed on the surface of the skin near the respiratory muscles [4]. Compared to its invasive counterpart, surface signal acquisition is an attractive alternative due to its practical approach and ease of use, and is suitable for a wide range of medical applications.

In general, the non-invasive recording of EMG activity is conducted by means of surface electrodes in a bipolar configuration. The main advantage of using a bipolar configuration lies in the ability to detect myographic activity on the surface by placing two electrodes on the muscle under study and then finding the difference between the sEMG recorded signals. This makes it the most straightforward and easy-to-use configuration for the clinical setting. However, unlike with non-respiratory muscles for which guidelines exist for electrode placement, interelectrode distance, and the orientation of the electrodes [5], no standardized electrode position guidelines for respiratory muscle sEMG recordings have yet been established. The literature points to different anatomical landmarks in various areas on the torso where respiratory muscle sEMG can be obtained, such as the lateral location on the rib cage (external intercostals) and the parasternal intercostal area [4]. However, factors such as posture, body composition or crosstalk from adjacent respiratory muscles may affect the sEMG recordings [6]. The bipolar configuration also has an associated spatial filtering effect that, in addition to the low-pass filtering effect of the volume conductor of tissues, leads to a smoothing of the sEMG signal. Therefore, the use of a bipolar configuration for the evaluation of a specific inner muscle such as the diaphragm is limited, as it results in poor spatial resolution. Furthermore, adjacent respiratory muscle activity and distant bioelectrical sources, such as electrocardiographic activity (ECG), interfere with the acquired sEMG signal, which makes studying it a challenge.

Extra-diaphragmatic respiratory muscles such as the sternocleidomastoid located in the neck are recruited when a load is imposed on the respiratory system [7]. Sternocleidomastoid muscle activity has shown potential in the diagnosis and assessment of chronic obstructive pulmonary disease patients [8]. Although the sternocleidomastoid is a prominent and a more accessible muscle, measuring its activity is affected by movement artefacts and/or crosstalk activity from surrounding muscles [9]. In particular, ECG affects trunk respiratory muscle sEMG signals due to the proximity of the heart and its great electrical intensity. Moreover, the ECG spectrum overlaps with that of the EMG, which hinders accurate interpretation. Consequently, filtering cardiac activity by means of classic methods involves the loss of respiratory muscle information [10]. From a signal processing standpoint, ECG contamination in sEMG signals can be suppressed through the application of subtraction [11], adaptive filtering [12], or entropy-based techniques [13]. Nonetheless, in addition to the high computational cost of the algorithms used, the performance of these techniques can be hampered when the subject has a high heart rate or when there are changes in the ECG morphology, as is the case with some cardiac diseases, which can limit its use in real-time applications. Alternatively, dedicated hardware has been proposed as a means of processing sEMG activity and removing cardiac contamination [14].

As a further step towards improving bipolar signal acquisitions, in the present work, we propose the use of concentric ring electrodes (CREs) as a more effective means of assessing inspiratory muscle function. CREs are a type of surface Laplacian electrodes that directly estimate the second spatial derivative of the potentials on the body's surface, acting as a filter that assigns more weight to the bioelectrical dipoles nearer to the electrode, providing more detail in distinguishing multiple bioelectric dipole sources [15]. CREs reduce mutual information present in bipolar signals picked up by disk electrodes and alleviate the related orientation problems [16], [17], [18]. CREs in different dimensions and configurations have been used in several bioelectric signal recording methods, such as electrocardiography [16], electroenterography [19], [20], electrohysterography [21], [22] and electromyography [23]. Specifically, preliminary studies have been conducted with diaphragmatic electromyography [24], [25].

The aim of this study conducted with healthy participants was therefore to investigate the performance of CREs in recording respiratory sEMG in the costal part of the diaphragm and sternocleidomastoid muscle during an inspiratory threshold loading test. In addition, we aimed to compare CRE measurements with those obtained simultaneously using the conventional surface bipolar configuration.

\section{Material AND Methods}

\section{A. Study Subjects}

Thirty-two healthy young adults (16 males and 16 females) with a mean (standard deviation) age of 23.5 (2.9) years, height of $1.70(0.08) \mathrm{m}$, weight of $63.8(11.8) \mathrm{kg}$, and body mass index of $21.9(2.7) \mathrm{kg} / \mathrm{m}^{2}$ with no relevant medical conditions volunteered to participate in this study. The signal database acquisition was conducted at the Biomedical Signal Processing and Interpretation Group at the Institute for Bioengineering of Catalonia (Barcelona, Spain) as approved by the Institutional Review Board committee (reference number IRB_IBEC_RJANE_2016_01). Participants were free to refuse to participate or to withdraw their consent at any point during the study. All participants were fully informed in detail of the study purpose, were familiarized with the procedures used, and gave written consent.

\section{B. Respiratory Protocol}

The participants performed six breathing tests: (1) a breathholding test, (2) a maximal inspiratory pressure (MIP) test and ( 3 to 6 ) four inspiratory load spontaneous breathing tests. This respiratory protocol was performed twice, switching the sEMG electrode arrangement to evaluate the influence of position in the comparison of electrode types. The order in which the two repetitions of the respiratory protocol were performed was random. 
(a)

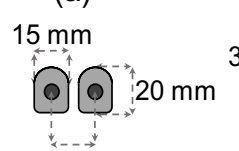

Inter-electrode

distance $20 \mathrm{~mm}$

conductive area

- electrode snap

\section{(b)}

$32 \mathrm{~mm}$

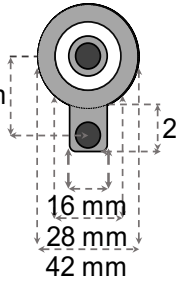

(c)

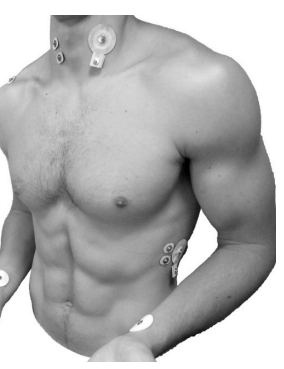

Fig. 1. Dimensions of (a) surface electrodes in bipolar configuration and (b) a concentric ring electrode (CRE) used for surface electromyographic recording on respiratory muscles. CRE is made up of a central disk and an external ring. (c) Electrode set-up for respiratory muscle signal acquisition, showing the bipolar configuration and CREs placed bilaterally in the lower chest close to the apposition zone of the diaphragm and in the sternocleidomastoid located in the neck (image shows the first electrode arrangement). Lead I ECG was recorded from upper limbs to detect the QRS of ECG signal. A refence electrode was in the right acromion.

An inspiratory muscle training device (Power Breathe ${ }^{\mathrm{TM}}$ KH2, IMT Technologies Ltd., Birmingham, England) was employed to perform the respiratory test. This portable handheld device applies resistance during inspiration via an electronically controlled valve which is opened once a target threshold pressure (set using the associated software) is reached (Breathe-Link Medic, version 1.0c, IMT Technologies Ltd., Birmingham, England). With this device, the global inspiratory muscle strength can be increased, and the MIP can be measured so that different levels of inspiratory load can be determined [24]. Over the course of the respiratory protocol, participants were asked to sit comfortably in a chair, hold themselves erect, and relax both arms on a table. A nose clip was provided to prevent nasal air leak.

\section{1) Breath-holding test}

Participants were asked to hold their breath just at the end of exhalation in a relaxed manner for about 15 to $20 \mathrm{~s}$. This maneuver ensures that respiratory muscle effort is minimized while background noise and cardiac activity are predominant, which enables the study of these undesired components of the sEMG.

\section{2) Maximal inspiratory pressure test}

An MIP test was performed as a volitional measure of respiratory muscle strength. The MIP involves the recruitment of the diaphragm and other inspiratory muscles and reflects their force-generating capacity [2]. Each participant was instructed to exhale to a residual volume followed by a maximal inspiratory effort. They were asked to perform the MIP maneuver at least five times with rests between attempts to obtain acceptable and reproducible inspiratory pressure measures. The highest value achieved was included in the study. During the test, participants were verbally encouraged to accomplish the maximal inspiratory effort.

\section{3) Spontaneous breathing tests}

Participants underwent four spontaneous breathing tests in which the inspiratory load on the respiratory system was increased. First, participants breathed without any load, that is, at quiet tidal breathing (QB). Then, the inspiratory load was increased to three different levels of inspiratory threshold loads, set at 20\%,40\% and 60\% of the MIP (L20\%, L40\% and L60\%, respectively) [24]. Participants took fifteen breaths for each loading test followed by five minutes of rest to recover and ensure that respiratory measures returned to baseline. They were encouraged to inhale forcefully and deeply followed by exhaling until their functional residual capacity was reached. Participants were free to choose their own pattern and timing of breathing (respiratory rate and fractional inspiratory time).

\section{Measurements and Data Acquisition \\ 1) Electrodes employed and skin preparation}

Respiratory sEMG signals were measured using two different electrode configurations: (1) a pair of surface electrodes (DENIS01520, Spes Medica, Battipaglia, Italy) in bipolar configuration with an inter-electrode distance of 20 $\mathrm{mm}$, and (2) a CRE (CODE5000S0, Spes Medica, Battipaglia, Italy). The CRE is made up of a central disk and an external ring. The dimensions of both surface electrodes are shown in Figure 1. Prior to the attachment of the surface electrodes, the subjects' skin was prepared by means of gentle local abrasion using an abrasive cream (Nuprep, Weaver and Company, Aurora, CO, USA) and swabbed with isopropyl alcohol to improve the skin-electrode impedance [26]. A reference electrode (DENIS01520, Spes Medica, Battipaglia, Italy) was attached to the skin near the right acromion of the scapula, which was taken as an electrically neutral zone.

\section{2) $s E M G$ of diaphragm muscle}

The surface EMG of the diaphragm muscle (sEMGdi) was measured bilaterally using both a bipolar configuration and a CRE placed on the skin of the lower chest near the apposition zone of the costal portion of the diaphragm, between the midaxillary and anterior axillary lines over the 7th and 8th intercostal spaces [27]. Different electrode arrangements were used in each of the two repetitions of the respiratory protocol. In the arrangement 1 , on the left side, the bipolar configuration was placed close to the anterior axillary line, followed by the CRE which was close to the mid-axillary line, as shown in Figure 1, while, on the right side, the CRE was more proximal to the anterior line and the bipolar configuration was closer to the mid-axillary line. In the arrangement 2 the position of the electrodes was interchanged.

\section{3) $s E M G$ of sternocleidomastoid muscle}

The electrical activity of the sternocleidomastoid muscle (sEMGsc) was measured using a pair of bipolar electrodes and a CRE placed bilaterally on the skin of the neck. On both sides, electrodes were positioned over the lower half of the sternocleidomastoid based on the palpation of the muscle [28]. Since this muscle is smaller and it is not possible to acquire both configurations at the same time on the same side, in this case in electrode arrangement 1 , the CRE was placed on the left side of the neck and the bipolar configuration was placed on the right side, as shown in Figure 1. Again, in electrode arrangement 2 the position of the electrodes was interchanged. 4) ECG and mouth pressure

In addition to the sEMG signals, ECG was recorded from a standard Lead I derivation using two $\mathrm{Ag} / \mathrm{AgCl}$ disk electrodes (11-mm diameter, pregelled, foam electrode 50/PK - EL501, BIOPAC Systems, Santa Barbara, CA, USA) placed on either wrist. Surface bioelectric measurements were taken relative to the same reference electrode, placed near the right acromion, as shown in Figure 1. Moreover, the mouth pressure signal 


\section{ELECTRODE ARRANGEMENT 1}

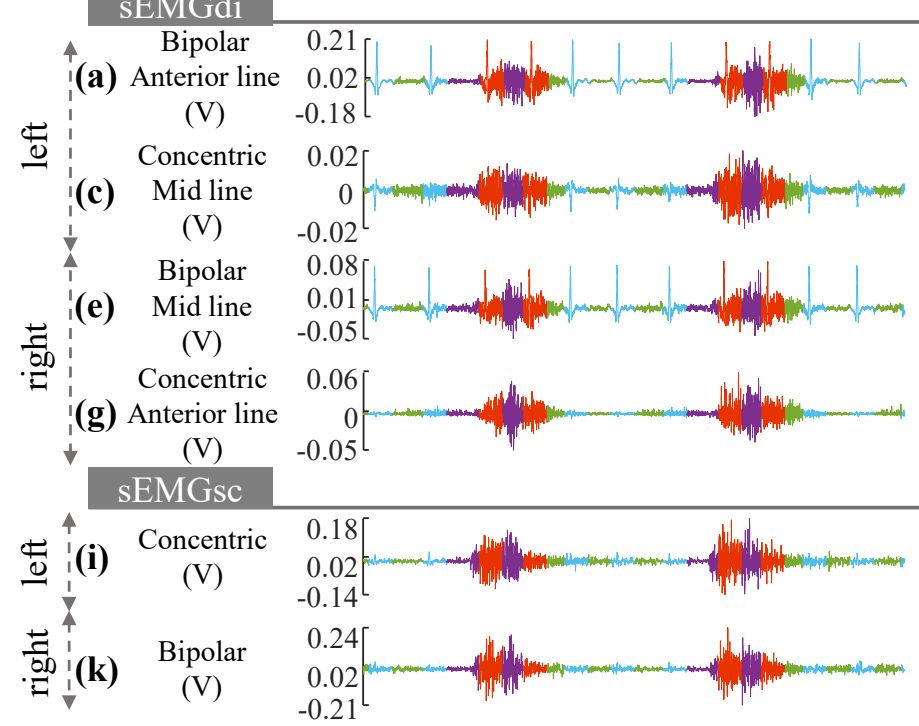

(m) $\begin{gathered}\text { Pmouth } \\ \left(\mathrm{cm} \mathrm{H}_{2} \mathrm{O}\right)\end{gathered}$

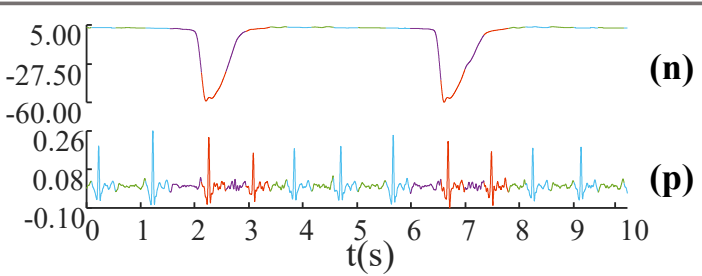

\section{ELECTRODE ARRANGEMENT 2}

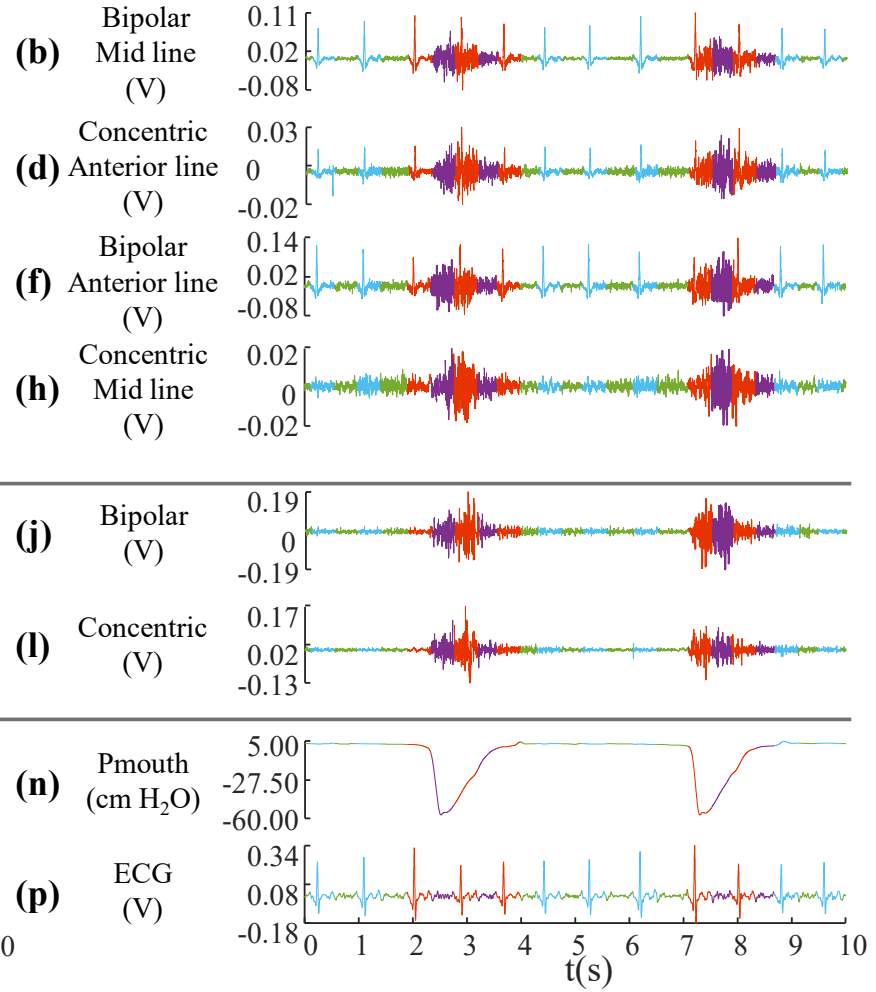

\footnotetext{
Inspiratory segment with cardiac activity (IWC) Inspiratory segment without cardiac activity (INC)
}

Expiratory segment with cardiac activity (EWC) Expiratory segment without cardiac activity (ENC)

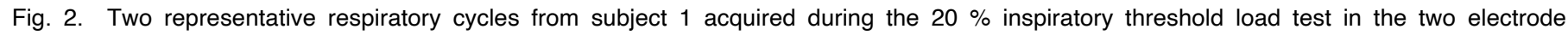

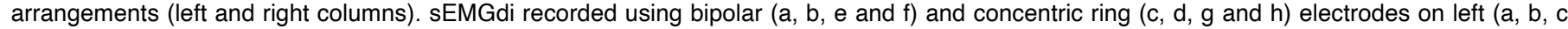

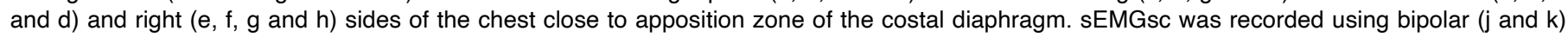

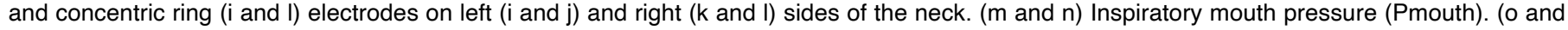
p) Lead I ECG.

(Pmouth) was measured with a differential pressure transducer (TSD160A BIOPAC Systems, Inc.).

\section{5) Signal Conditioning and Acquisition}

sEMG signals were conditioned using modular differential amplifiers (EMG100C, BIOPAC Systems, Inc.) with a gain of 1000 and an analogue band-pass filter with cut-off frequencies between $1 \mathrm{~Hz}$ and $500 \mathrm{~Hz}$. ECG was conditioned using a modular differential amplifier (ECG 100C, BIOPAC Systems Inc.) with a gain of 2000 and an analogue band-pass filter with cut-off frequencies of $0.5 \mathrm{~Hz}$ and $35 \mathrm{~Hz}$. Pmouth was conditioned using a modular differential amplifier (DA100C, BIOPAC Systems, Inc.) with a gain of 1000 and an analogue low-pass filter with a cut-off frequency of $300 \mathrm{~Hz}$. All signals were displayed in real time on a monitor and saved to the computer via the accompanying software (AcqKnowledge software, v.3.2, BIOPAC Systems Inc.) and sampled at 2000 $\mathrm{Hz}$ using a 16-bit analog-to-digital converter data acquisition system (MP150, Biopac Systems Inc.).

Figure 2 shows an example of the acquired signals during the $20 \%$ inspiratory threshold load test using the two electrode arrangements.

\section{Signal Analysis}

1) Preprocessing

Respiratory sEMG signals were filtered with a fourth order, zero-phase Butterworth bandpass filter with cut-off frequencies of 5 and $400 \mathrm{~Hz}$. Fundamental power line interference of $50 \mathrm{~Hz}$ and its harmonics were removed using an infinite impulse response comb filter.

\section{2) Segmentation}

To assess respiratory muscle activity, only the inspiratory segments of the signal must be examined, without considering heart activity, as it would bias the evaluation. Inspiratory and expiratory cycles were segmented by detecting the onset and offset time instants of consecutive breathing cycles from the Pmouth signal using a threshold-based criterion [29]. From these onset and offset detections, inspiratory time, fractional inspiratory time, and respiratory rate were calculated. The QRS complexes from the ECG signal were detected using the classical Pan-Tompkins algorithm [30]. The start and end of the cardiac activity were considered $150 \mathrm{~ms}$ before and 300 $\mathrm{ms}$ after the $\mathrm{R}$ point of the QRS complex, respectively. Considering all the time instants, the respiratory signals were classified into four segment types, as shown in Figure 2: (1) inspiratory with cardiac activity (IWC), (2) expiratory with 


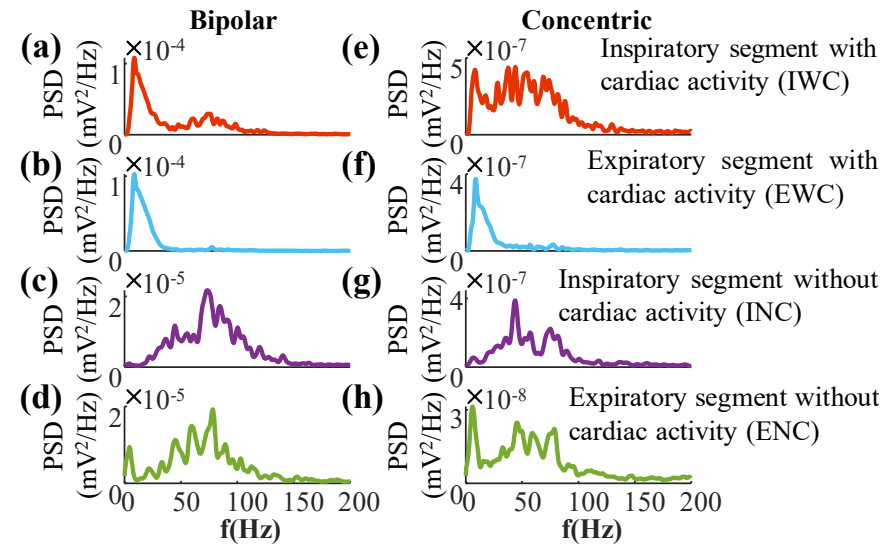

Fig. 3. Weighted average power spectral density (PSD) corresponding to the four segment types for the sEMGdi signal recorded on the left side of the chest using the bipolar configuration placed close to the anterior axillary line (left column) and a concentric ring electrode placed close to the mid-axillary line (right column) from subject 1 , from subject 1 acquired during the $20 \%$ inspiratory threshold load test using the electrode arrangement 1 . (a and e) PSD of the inspiratory with cardiac activity (IWC), (b and f) PSD of the expiratory with cardiac activity (EWC), (c and g) PSD of the inspiratory without cardiac activity (INC) and ( $d$ and h) PSD of expiratory without cardiac activity (ENC).

cardiac activity (EWC), (3) inspiratory without cardiac activity (INC) and (4) expiratory without cardiac activity (ENC). In the breath-holding maneuver, signals were also divided into two segment types: (1) with cardiac activity (BHWC) and (2) without cardiac activity (BHNC). Respiratory sEMG signals containing artifacts originating from breathing or movement in any of the respiratory tests were discarded from the analysis.

\section{3) Power spectral density estimation}

First, the power spectral density (PSD) of each segment of all the sEMG signals was estimated using the modified periodogram method with a Hamming window and a discrete Fourier transform with 4096 points [24], [31]. Then, a weighted average PSD of all the single PSDs corresponding to segments of the same type was calculated and the spectral power (P) and the median frequency (MDF) of each type of segment were computed. In addition, the P of the INC-type segments $\left(\mathrm{P}_{\mathrm{INC}}\right)$ were normalized to the maximal $\mathrm{P}$ of the INCtype obtained during the MIP test ( $\left.\mathrm{P}_{\mathrm{INC}} \% \mathrm{max}\right)$.

Figure 3 shows an example of the average PSD of every type of segment corresponding to sEMGdi signals recorded in the left side of the chest using a bipolar and a CRE configuration from the electrode arrangement 1 showed in Figure 2.

\section{4) Power ratios from SEMG signals acquired during the respiratory protocol}

Three power ratios were calculated for each load. The purpose of the first ratio ( $\mathrm{R}_{\text {ins/noise }}$ ) was to evaluate the inspiratory sEMG activity in relation to the basal activity recorded in the breath-hold test (noise). Therefore, the $\mathrm{P}$ of the INC-type segments were related to the P of the BHNC-type segments acquired during the breath-hold test in accordance with the following equation:

$$
R_{\text {ins } / \text { noise }}(d B)=10 \log \frac{P_{I N C}}{P_{B H N C}}
$$
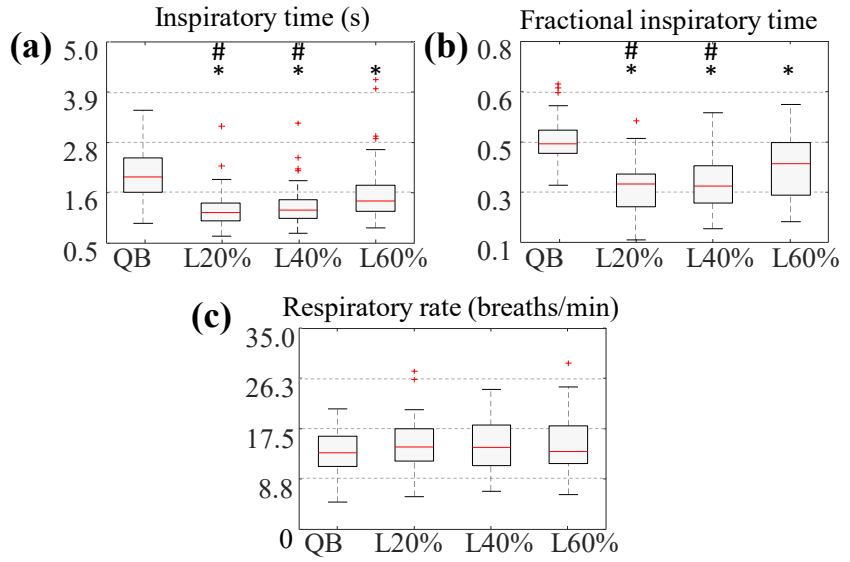

Fig. 4. Box-whisker plots of the (a) inspiratory time (b) fractional inspiratory time and (c) respiratory rate during the unloaded and loaded breathing of the respiratory protocol. All data points denoted with $\left(^{*}\right)$ and (\#) symbols were significantly different from QB and L60\%, respectively. Statistically significant differences were set at $\mathrm{p}<0.05$.

The second ratio $\left(\mathrm{R}_{\text {ins/cardio }}\right)$ was created to appraise the inspiratory sEMG activity in relation to heart activity interference, and considers the $\mathrm{P}$ of the INC-type segments in relation to the $\mathrm{P}$ of the BHWC-type segments:

$$
R_{\text {ins/cardio }}(d B)=10 \log \frac{P_{\text {NCC }}}{P_{B H W C}}
$$

Finally, the purpose of the third ratio $\left(\mathrm{R}_{\text {ins/exp }}\right)$ was to evaluate the inspiratory sEMG activity in relation to expiratory activity. Therefore, for this ratio, the $\mathrm{P}$ of the INCtype segments was related to the P of the ENC-type segments, in accordance with the following equation:

$$
R_{i n s / \exp }(d B)=10 \log \frac{P_{I N C}}{P_{E N C}}
$$

\section{E. Data analysis}

We first checked the possible influence of the relative positions of the recording sites and the consistency of the sEMG parameters between the two acquisitions. A nonparametric MANOVA test showed no statistically significant difference $(\mathrm{p}>0.05)$ between $\mathrm{sEMGdi}$ parameters recorded in the anterior and posterior positions for any recording site (left, right), configuration (concentric, bipolar) or respiratory load (QB, 20\%, 40\% and 60\%). sEMGdi parameters from both relative positions were combined in subsequent analyses to facilitate the comparison between electrode configurations. For each type of electrode (bipolar and CRE), position (left and right) and muscle (diaphragm and sternocleidomastoid), a Friedman's non-parametric test was used to assess significant differences in each sEMG parameter among the group of the four respiratory loads (load comparison). A two-sided Wilcoxon signed-rank test was used for pairwise comparison of parameters between loads, for left vs. right (side comparison) and for bipolar vs. concentric electrode (configuration comparison). A two-sided Wilcoxon rank sum test was used when comparing sEMGdi vs. sEMGsc (muscle comparison) parameters for a given electrode configuration and recording side. 


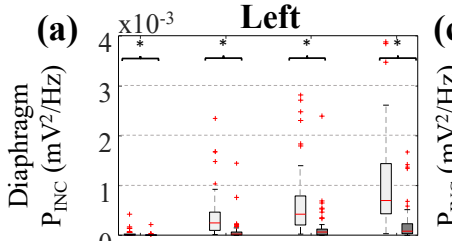

(b) $0 \stackrel{\mathrm{QB}}{\mathrm{QB}} \mathrm{L} 20 \% \mathrm{~L} 40 \% \quad \mathrm{~L} 60 \%$
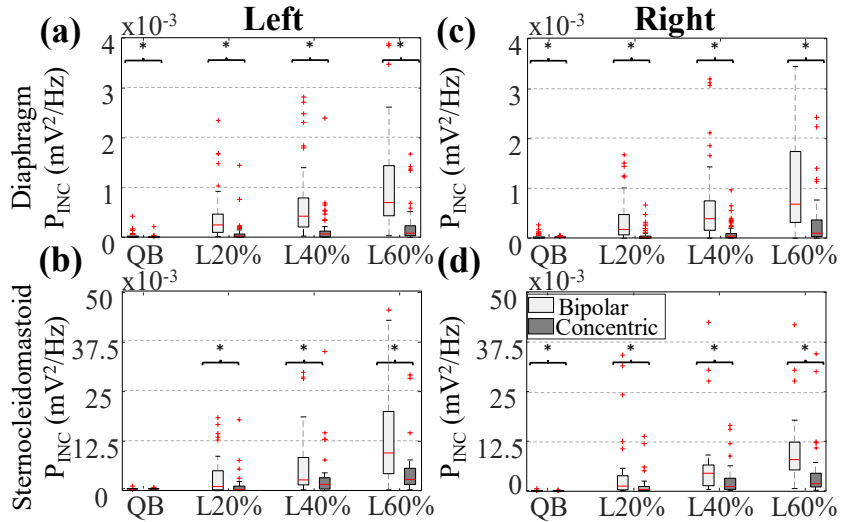

Fig. 5. Box-whisker plots of the spectral power of inspiratory segments without cardiac activity ( $P_{\text {INC }}$ ) for the different levels of inspiratory loads of electrode configurations (bipolar, concentric). (a) sEMGdi and (b) sEMGsc recorded on the left side. (c) sEMGdi and (d) sEMGsc recorded on the right side. Statistically significant differences $(p<0.05)$ between electrode configurations are shown $\left(^{*}\right)$.

A significance level of $p=0.05$ was established for all statistical tests. All offline analyses were performed using MATLAB (MathWorks, Inc., v. R2019b, Natick, MA, USA).

\section{REsults}

Figure 4 shows the distribution (Box-whisker plots) of inspiratory time, fractional inspiratory time, and respiratory rate during the evolution of the respiratory protocol. Inspiratory time and fractional inspiratory time at unloaded breathing $(\mathrm{QB})$ were significantly different when compared to loaded breathings (L20\%, L40\% and L60\%). Significant differences were also found between $\mathrm{L} 60 \%$ and $\mathrm{L} 20 \%$ and L40\%. Inspiratory loading did not significantly change respiratory rate during the respiratory protocol.

Figures 5 to 10 show the distribution (Box-whisker plots) of the computed parameters from sEMGdi and sEMGsc on both sides of the body for the different respiratory loads of the spontaneous breathing tests (QB, L20\%, L40\%, L60\%) and electrode configurations (bipolar, concentric). Statistically
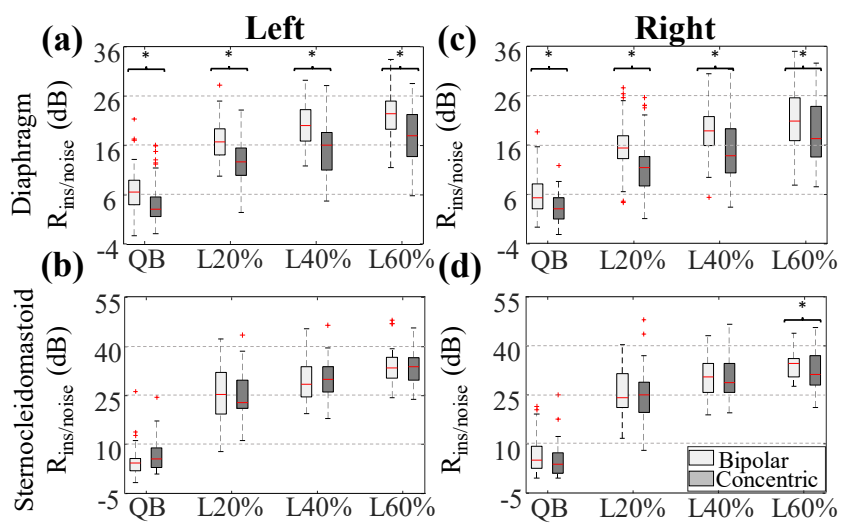

Fig. 7. Box-whisker plots of power ratio of inspiratory sEMG activity in relation to the power of basal sEMG without ECG (Rins/noise) for the different levels of inspiratory loads of electrode configurations (bipolar, concentric). (a) sEMGdi and (b) sEMGsc recorded on the left side. (c) sEMGdi and (d) sEMGsc recorded on the right side. Statistically significant differences $(p<0.05)$ between electrode configurations are shown $\left({ }^{*}\right)$.
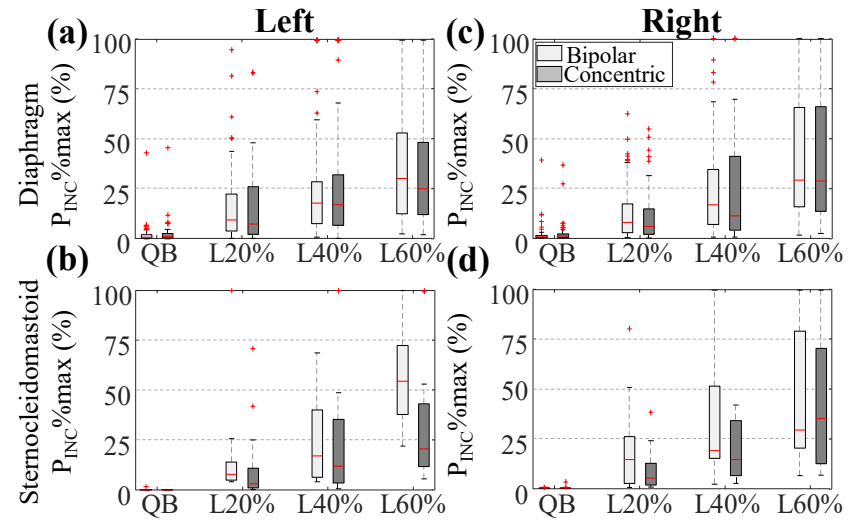

Fig. 6. Box-whisker plots of the spectral power of inspiratory segments without cardiac activity $\left(P_{\text {INC }}\right)$ normalized to maximal $P_{\text {INC }}$ ( $P_{\text {INC\% }}$ max) during maximal inspiratory maneuver for the different levels of inspiratory loads of electrode configurations (bipolar, concentric). (a) sEMGdi and (b) sEMGsc recorded on the left side. (c) sEMGdi and (d) sEMGsc recorded on the right side. No statistically significant differences $(p>0.05)$ were found between electrode configurations.

significant results of the comparisons of electrode configurations are shown within the figures, while, for the sake of simplicity and clarity, the main load, side, and respiratory muscle results are described throughout the text.

The results of $\mathrm{P}_{\mathrm{INC}}$ and $\mathrm{P}_{\mathrm{INC}} \% \max$ are presented in Figure 5 and 6 , respectively. $\mathrm{P}_{\mathrm{INC}}$ of the bipolar configuration is statistically different from the $\mathrm{P}_{\mathrm{INC}}$ of the concentric configuration in all cases (except for sEMGdi at QB; $\mathrm{p}=$ 0.059), with greater values for the bipolar configuration. However, the comparison of the $\mathrm{P}_{\mathrm{INC}} \%$ max did not reveal statistically significant differences between bipolar and CRE configurations in both muscles at any load or side (Figure 6). In both cases, absolute and normalized, the greater the respiratory load, the greater the signal power for the diaphragm and sternocleidomastoid muscles on both sides and for both electrode configurations. For each respiratory load and electrode configuration, the signal power was of the same order of magnitude, with no statistically significant differences
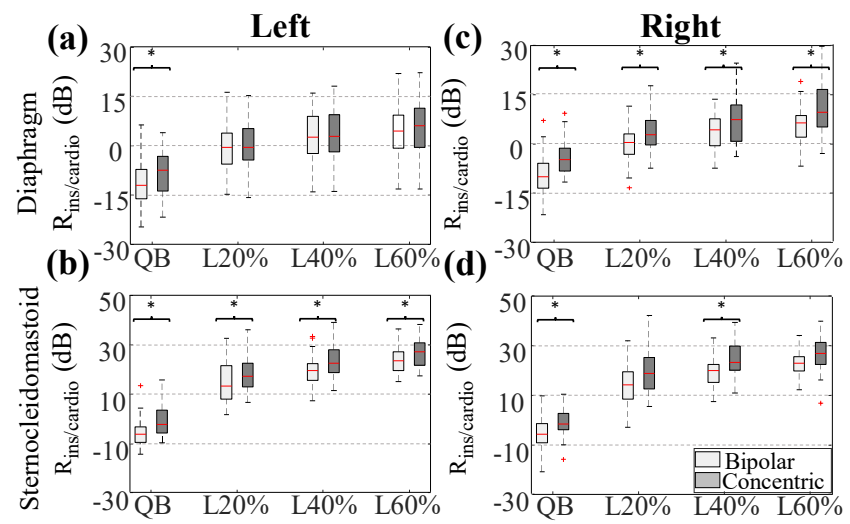

Fig. 8. Box-whisker plots of power ratio of inspiratory sEMG activity in relation to the power of basal SEMG with ECG (Rins/cardio) for the different levels of inspiratory loads of electrode configurations (bipolar, concentric). (a) sEMGdi and (b) sEMGsc recorded on the left side. (c) sEMGdi and (d) sEMGsc recorded on the right side. Statistically significant differences $(p<0.05)$ between electrode configurations are shown $\left(^{*}\right)$. 

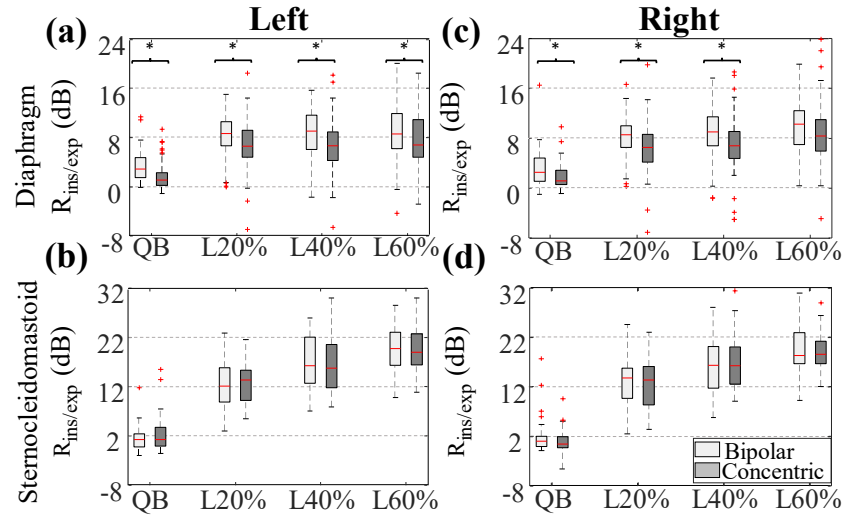

Fig. 9. Box-whisker plots of power ratio of inspiratory sEMG activity in relation to the power of expiratory sEMG without ECG (Rins/exp) for the different levels of inspiratory loads of electrode configurations (bipolar, concentric). (a) sEMGdi and (b) sEMGsc recorded on the left side. (c) sEMGdi and (d) sEMGsc recorded on the right side. Statistically significant differences $(p<0.05)$ between electrode configurations are shown $\left(^{*}\right)$.

between sides. The signal power of sEMGsc was significantly greater than that of sEMGdi for every load except for QB when using CREs.

We found significant differences in $\mathrm{R}_{\text {ins/noise }}$ (Figure 7), with higher values obtained for the bipolar configuration than the concentric configuration in the case of the diaphragm. However, no significant differences between the bipolar and concentric configurations were found on $\mathrm{R}_{\text {ins/noise }}$ at any load or side for the sternocleidomastoid (except for L60\% on the right side). The effects of respiratory load, muscle and recording side showed very similar trends to $\mathrm{P}_{\mathrm{INC}}$ (Figure 5): increasing values of $\mathrm{R}_{\text {ins/noise with the respiratory load, higher }}$ values in sEMGsc than in sEMGdi and similar values on both sides.

Figure 8 shows the results of the signal to cardiac interference ratio $\left(\mathrm{R}_{\text {ins/cardio }}\right)$. The comparison of the electrode configuration revealed that the $\mathrm{R}_{\text {ins/cardio }}$ of the concentric configuration was greater than that of the bipolar set-up, with significant differences in all cases, except for sEMGdi on the left side at forced inspiratory load levels and two loads of sEMGsc (right side L20\% and L60\%, with $\mathrm{p}=0.052$ and $\mathrm{p}=$ 0.063 , respectively). The greater signal power in higher respiratory loads becomes an increase in $\mathrm{R}_{\text {ins/cardio }}$ for both muscles and recording sides. $\mathrm{R}_{\text {ins/cardio }}$ is also significantly greater in sEMGsc than in sEMGdi. Significant differences in relation to the influence of recording side were only found for sEMGdi acquired with CREs for all loads, with greater values on the right side.

The results of $\mathrm{R}_{\text {ins/exp }}$ are displayed in Figure 9. Our assessment of the influence of electrode configuration found significantly greater values of $\mathrm{R}_{\text {ins/exp }}$ for bipolar than for concentric sEMGdi (except for L60\% on the right side, $\mathrm{p}=$ 0.1 ), and no significant differences were found at any load or side for sEMGsc. We found a significant increase in $\mathrm{R}_{\text {ins/exp }}$ from QB to forced inspiratory loads in both muscles and electrode configurations. In the case of sEMGdi, the $\mathrm{R}_{\text {ins/exp }}$ values showed an almost constant trend when increasing forced respiratory load and no significant differences between
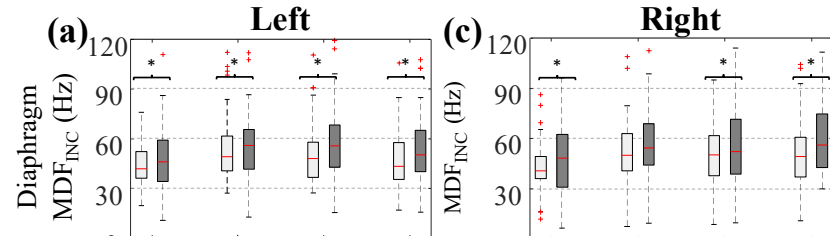

(b) 0 QB $\quad$ L20\% $\quad$ L40\% $\quad$ L60\%

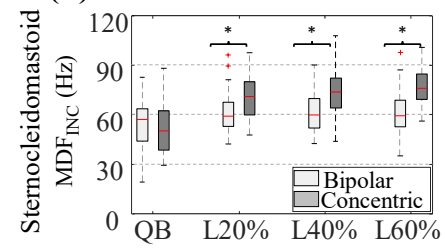

(d) $0 \quad$ QB $\quad$ L20\% $\quad$ L $40 \% \quad \mathrm{~L} 60 \%$

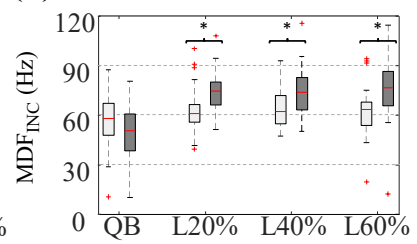

Fig. 10. Box-whisker plots of median frequency during inspiration without ECG $\left(\right.$ MDF $\left._{\mathrm{INC}}\right)$ for the different levels of inspiratory loads of electrode configurations (bipolar, concentric). (a) sEMGdi and (b) sEMGsc recorded on the left side. (c) sEMGdi and (d) sEMGsc recorded on the right side. Statistically significant differences $(p<0.05)$ between electrode configurations are shown $\left(^{*}\right)$.

any forced inspiratory load levels. In the case of sEMGsc, an increasing trend was observed with the inspiratory load, with significant differences between L20\% and L60\%. The $\mathrm{R}_{\text {ins/exp }}$ from sEMGsc was significantly greater than sEMGdi when an inspiratory load was imposed. The results did not reveal any remarkable influence of recording side.

Figure 10 shows the $\mathrm{MDF}_{\mathrm{INC}}$ results. Greater $\mathrm{MDF}_{\mathrm{INC}}$ values were observed for CREs than for the bipolar configuration in both respiratory muscles, most of them statistically significant. Smaller $\mathrm{MDF}_{\mathrm{INC}}$ values in $\mathrm{CRE}$ recordings were only found in sEMGsc at QB. It is noteworthy that, in terms of the effect of the inspiratory load, we found a significant increase in $\mathrm{MDF}_{\mathrm{INC}}$ from QB to any forced inspiratory load in both muscles and recording sites in the case of CREs. For bipolar recordings, significant differences from QB to forced inspiratory loads were found in very few and specific cases of sEMGdi and in no case of sEMGsc. Regarding muscle comparison, the $\mathrm{MDF}_{\mathrm{INC}}$ of sEMGsc was greater than that of sEMGdi with significant differences in all cases except at QB with CREs. The results did not reveal any remarkable influence of recording side.

\section{DISCUSSION}

In the present work, sEMG signals from the diaphragm and sternocleidomastoid muscles were recorded during an inspiratory loading threshold test via traditional bipolar electrodes and CREs. The performance of CREs was compared to that of electrodes in a bipolar configuration under different conditions. The respiratory sEMG signals were characterized, and the computed parameters were compared considering aspects such as respiratory load, muscle, side and, especially, electrode configuration.

\section{A. Evaluation of SEMG recordings with CRES}

The signal power of the CRE recordings of the sEMG respiratory muscles was weaker than that of the bipolar recordings. This is consistent with previously reported results on other bioelectrical recordings such as ECG [18], electroenterogram (EEnG) [19] and electrohysterogram (EHG) [22]. This is attributed to the enhanced spatial resolution of 
Laplacian recordings, which makes the volume of sensed bioelectric dipoles smaller and thus the amplitude of the captured signal smaller as well. Another factor that can significantly influence the amplitude and quality of the signal recorded with CRE is the electrode placement. Since the volume of sensed bioelectric dipoles is smaller than with conventional bipolar and does not follow muscle fiber orientation, electrode placement can thus be more relevant. If the CRE is not placed precisely over the motor unit, a smaller signal will be captured, and quality will be reduced. Moreover to date, there is a lack of consensus concerning the placement of the surface electrodes for the respiratory muscles activity recording [4]. In this study, anatomical landmarks were used for the sEMG recording of diaphragm [42], [27], [40] and sternocleidomastoid [40], [28], [8] as done in previous works.

The influence of the electrode configuration on the signalto-noise ratio depends on the respiratory muscle whose activity is recorded. In sEMGdi, the ratio was lower for the concentric configuration, whereas no significant differences were found in the sternocleidomastoid recordings. Previous works also found discrepancies in the effect of the electrode configuration on signal quality parameters depending on the muscle recorded: signal-to-noise ratios of Laplacian estimates lower than those from conventional recording with disk electrodes have been reported for ECG [32] and sEMG of biceps brachii [33], while no differences were obtained in sEMG from the forearm [34] or from the uterine muscle [22]. These works also showed that the signal quality ratio of signals recorded via CREs varies depending on the two main issues that affect the spatial resolution of the Laplacian estimate: the inter-ring dimension and the CRE configuration (bipolar, tripolar). In this work, the central disk diameter is 16 $\mathrm{mm}$ and that of the ring median line is $35 \mathrm{~mm}$. Greater interring distances could yield higher quality signals, although with poorer spatial resolution [22], [35]. Concentric sEMG recording remarkably reduced the interference of cardiac activity in comparison to bipolar recordings. This is especially relevant since, as can be deduced from the lower values of $\mathrm{R}_{\text {ins/cardio }}$ versus $\mathrm{R}_{\text {ins/noise }}$, the power of cardiac interference is of a greater magnitude than that of background noise. The better spatial resolution and greater far-field activity attenuation of CREs [36] have been proven to reduce cardiac activity interference in uterine [37] and intestinal sEMG recordings [19], [20]. Still, this attenuation depends on the distance between the activity wavefront and the center of the sensor. A bipolar configuration with disk electrodes enhances localized information by rejecting the far field with the inverse third power of distance, whereas in the CRE configuration used in this study, far-field rejection is proportional to the inverse fourth power of the distance [36]. The electrode location on the left of the diaphragm is very close to the heart. The difference in the attenuation of the far-field cardiac activity between the two electrode configurations is therefore lesser in this position. This agrees with the lesser differences found in the signal-to-cardiac interference ratio of concentric and bipolar sEMGdi of the left side in forced respiratory loads. Still, differences were significant on the right side of the diaphragm and on the sternocleidomastoid, which are farther from the heart, and in quiet breathing in all cases.
sEMGdi recorded with bipolar configuration yielded greater inspiration-to-expiration power ratio values than those recorded via the CRE configuration, whereas no differences were found in sEMGsc. This may be because the sternocleidomastoid is a more superficial muscle than the diaphragm, and CREs are less sensitive to deeper dipole sources [36].

Higher median frequency values were obtained in concentric sEMG and the differences with the bipolar configuration were greater in the sternocleidomastoid. This points to a lower attenuation of the high-frequency content of concentric SEMG signals, which is consistent with theoretical studies that show that the concentric configuration has a greater high-pass filtering effect than conventional bipolar recording [38]. This spatial filtering depends on the depth of the bioelectric dipoles, and its effects are more pronounced in muscles near the surface, as is the case of the sternocleidomastoid muscle. Higher median frequency values and wider frequency bandwidth for Laplacian signals have been found in sEMG of biceps brachii [33] and mean frequency values on uterine muscle [22]. The capability of Laplacian recordings to pick up sEMG components of higher frequencies could provide a signal bandwidth closer to that of the intramuscular recordings obtained with needle electrodes [39] and enhance the fatigue monitoring of the respiratory muscle, as reported for biceps [33]. In this study, differences in MDF between quiet tidal breathing and inspiratory loads were only significant with concentric recordings. Therefore, CREs could potentially enhance the study of shifts in the frequency content of the respiratory sEMG by providing more information about activated muscle fibers for the evaluation of respiratory muscles in comparison to the bipolar configuration.

\section{B. Effect of increasing respiratory load}

sEMG signal power, absolute and normalized, and both $\mathrm{R}_{\text {ins/noise }}$ and $\mathrm{R}_{\text {ins/cardio }}$ signal quality parameters increased with increasing respiratory load for the diaphragm and the sternocleidomastoid. These results agree with previous works in which a load was imposed on the respiratory system and electrical activity was non-invasively recorded on different respiratory muscles: diaphragm [27], [40], [41], [42], sternocleidomastoid [8], scalene and parasternal [42], and in healthy subjects [27], [40], [41], [42] and COPD patients [8], [40], [42]. In all these studies, the respiratory electromyographic activity was recorded using a conventional approach, that is, with disk electrodes in a bipolar configuration. The present study shows that this behavior is consistent when sEMG is recorded via CREs.

In our study, the increase in the $\mathrm{R}_{\text {ins/exp }}$ of the diaphragm muscle was only significant during the transition from quiet unloaded breathing to respiratory loads. In contrast to power and $\mathrm{R}_{\text {ins/noise, }}$, there were no significant changes in this ratio between $\mathrm{L} 20 \%, \mathrm{~L} 40 \%$ and $\mathrm{L} 60 \%$, which demonstrates that expiratory activity increases with inspiratory loads and proportionally to that of inspiratory activity. This agrees with that observed in [43], in which mechanical loads applied only during inspiration were found to influence the behavior of the 
respiratory musculature during exhalation. Again, this is consistent when sEMG is recorded via CREs.

The same trend was observed for the frequency domain analysis in which we observed a shift in the respiratory sEMG PSD towards higher frequencies as the force generation capacity on the respiratory muscles increased, as reflected by the MDF. Similar behavior was reported in [44]. The need for extra respiratory effort when an additional inspiratory load is imposed requires the recruitment of motor units with fasttwitch muscle fibers resulting in an increase in MDF [45]. However, within the different load levels, the increase in muscular activity is only reflected in an increase in signal power, without a notable frequency shift during the respiratory protocol. This evolution of sEMGsc frequency is consistent with findings from another study using a bipolar configuration to record sEMGsc in chronic obstructive pulmonary disease patients at increasing inspiratory loads [8]. They found a sudden increase in MDF from quiet breathing to the first inspiratory load and the following increases in loads reflected almost constant values. The changes of sEMG PSD towards higher frequencies were more evident when evaluating the sEMGdi and sEGMsc signals recorded by CREs compared to the bipolar configuration. The improved sensing of high frequency components by means of CREs compared to conventional bipolar recordings has been reported in other bioelectric signals such as EEG [46].

\section{Diaphragm and sternocleidomastoid sEMG}

sEMGsc was proven to have greater inspiratory signal power, $\mathrm{R}_{\text {ins/noise, and }} \mathrm{R}_{\text {ins/exp }}$ than sEMGdi at all inspiratory loads. One explanation for this is that the sternocleidomastoid is a more accessible and superficial muscle that allows surface signals of greater amplitude to be captured than in the case of the diaphragm. Our findings are consistent with those reported in [7] and [40] in which sEMGsc activity was higher than sEMGdi. Only during quiet tidal breathing were the power and ratios of sEMGsc and sEMGdi similar. This is because the sternocleidomastoid is an auxiliary respiratory muscle which is recruited when an inspiratory load is imposed on the respiratory system [7].

The location of the diaphragm also makes sEMGdi more prone to the effects of cardiac interference than sEMGsc, yielding poorer signal-to-cardiac interference ratios $\left(\mathrm{R}_{\text {ins/cardio }}\right)$. In fact, only in sEMGdi, with an expected greater cardiac interference on the left side, was a significant influence on the registration side found when using CREs. The higher values of the median frequency in sEMGsc suggest that in this more superficial muscle the low-pass filtering effect between the myoelectric activity captured on the surface and that generated by the muscle [47] is reduced. Also, these higher MDF values in sEMGsc could be due to the fact that the proportion of fasttwitch fibers in the sternocleidomastoid muscle is higher than in the diaphragm. It has been reported that $65 \%$ of muscle fibers in the sternocleidomastoid are fast-twitch fibers [48]. EMGdi represents the global activity of the costal portion of the diaphragm and the lower intercostal muscles. The proportion of fast-twitch fibers in the costal diaphragm and intercostal muscles is only $50 \%$ and $40 \%$, respectively [49]. Again, the differences between sEMGdi and sEMGsc in the different parameters mentioned above were consistent in the recordings taken using both bipolar and concentric electrodes.

\section{CONCLUSION}

Respiratory muscle sEMG analysis can benefit from the advantages that the concentric electrode configuration has over the bipolar with disk electrodes set-up, such as the attenuation of bioelectrical interferences (such as the ECG) and picking up EMG components of higher frequencies. In addition, the placement of CREs is simpler as they behave in the same manner in all directions and do not have to be oriented in accordance with the muscle fibers as with the bipolar configuration; this is particularly relevant in respiratory muscles such as the diaphragm in which identifying the orientation of the muscle fibers pose a challenge.

CREs have great potential for improving clinical practice in the monitoring and evaluation of respiratory muscle activity. Future work on subjects with a wider range of age and body mass index and pathological subjects would be necessary to further assess the possible advantages of using CREs for sEMG recording in respiratory disease monitoring and clinical diagnosis. It would also be interesting to test other CRE configurations with a greater number of rings and with different distances between rings, which could show an improvement in the estimation of the Laplacian potential.

\section{REFERENCES}

[1] A. J. Lopes, "Advances in spirometry testing for lung function analysis," Expert Rev. Respir. Med., vol. 13, no. 6, pp. 559-569, Apr. 2019.

[2] P. Laveneziana et al., "ERS statement on respiratory muscle testing at rest and during exercise," Eur. Respir. J., vol. 53, no. 6, p. 1801214, Jun. 2019.

[3] C. J. Jolley et al., "Neural respiratory drive in healthy subjects and in COPD," Eur. Respir. J., vol. 33, no. 2, pp. 289-297, Feb. 2009.

[4] I. M. M. Dos Reis, D. G. Ohara, L. B. Januário, R. P. Basso-Vanelli, A. B. Oliveira, and M. Jamami, "Surface electromyography in inspiratory muscles in adults and elderly individuals: A systematic review," $J$. Electromyogr. Kinesiol., vol. 44, pp. 139-155, Feb. 2019.

[5] M. Beretta Piccoli et al., "Innervation zone locations in 43 superficial muscles: Toward a standardization of electrode positioning," Muscle Nerve, vol. 49, no. 3, pp. 413-421, Mar. 2014.

[6] C. J. De Luca, "The use of surface electromyography in biomechanics," J. Appl. Biomech., vol. 13, no. 2, pp. 135-163, Jan. 1997.

[7] A. H. Ramsook et al., "Diaphragm recruitment increases during a bout of targeted inspiratory muscle training," Med. Sci. Sports Exerc., vol. 48, no. 6, pp. 1179-1186, Jun. 2016.

[8] M. A. Mañanas, R. Jané, J. A. Fiz, J. Morera, and P. Caminal, "Study of myographic signals from sternomastoid muscle in patients with chronic obstructive pulmonary disease," IEEE Trans. Biomed. Eng., vol. 47, no. 5, pp. 674-681, May 2000.

[9] A. De Troyer, R. Peche, J.-C. Yernault, and M. Estenne, "Neck muscle activity in patients with severe chronic obstructive pulmonary disease," Am. J. Respir. Crit. Care Med., vol. 150, no. 1, pp. 41-47, Jul. 1994.

[10] J. D. M. Drake and J. P. Callaghan, "Elimination of electrocardiogram contamination from electromyogram signals: An evaluation of currently used removal techniques," J. Electromyogr. Kinesiol., vol. 16, no. 2, pp. 175-187, Apr. 2006.

[11] R. Bloch, "Subtraction of electrocardiographic signal from respiratory electromyogram," J. Appl. Physiol., vol. 55, no. 2, pp. 619-623, Aug. 1983.

[12] G. Lu et al., "Removing ECG noise from surface EMG signals using adaptive filtering," Neurosci. Lett., vol. 462, no. 1, pp. 14-19, Oct. 2009.

[13] L. Estrada, A. Torres, L. Sarlabous, and R. Jané, "Influence of parameter selection in fixed sample entropy of surface diaphragm electromyography for estimating respiratory activity," Entropy, vol. 19, 
no. 9, p. 460, Sep. 2017.

[14] H. F. R. Prechtl, L. A. Van Eykern, and M. J. O’Brien, "Respiratory muscle EMG in newborns: a non-intrusive method," Early Hum. Dev., vol. 1, no. 3, pp. 265-283, Dec. 1977.

[15] B. He and R. J. Cohen, "Body surface Laplacian ECG mapping," IEEE Trans. Biomed. Eng., vol. 39, no. 11, pp. 1179-1191, Nov. 1992.

[16] W. Besio and T. Chen, "Tripolar Laplacian electrocardiogram and moment of activation isochronal mapping," Physiol. Meas., vol. 28, no. 5, pp. 515-29, May 2007.

[17] K. Koka and W. G. Besio, "Improvement of spatial selectivity and decrease of mutual information of tri-polar concentric ring electrodes," J. Neurosci. Methods, vol. 165, no. 2, pp. 216-222, Sep. 2007.

[18] G. Prats-Boluda, Y. Ye-Lin, J. Bueno-Barrachina, R. Rodriguez de Sanabria, and J. Garcia-Casado, "Towards the clinical use of concentric electrodes in ECG recordings: influence of ring dimensions and electrode position," Meas. Sci. Technol., vol. 27, no. 2, pp. 1-11, Jan. 2016.

[19] J. Garcia-Casado, V. Zena-Gimenez, G. Prats-Boluda, and Y. Ye-Lin, "Enhancement of non-invasive recording of electroenterogram by means of a flexible array of concentric ring electrodes," Ann. Biomed. Eng., vol. 42, no. 3, pp. 651-660, Mar. 2014.

[20] V. Zena-Giménez, J. Garcia-Casado, Y. Ye-Lin, E. Garcia-Breijo, and G. Prats-Boluda, "A flexible multiring concentric electrode for noninvasive identification of intestinal slow waves," Sensors, vol. 18, no. 2, p. 396, Jan. 2018.

[21] G. Li et al., "Active Laplacian electrode for the data-acquisition system of EHG," in J. Phys.: Conf. Ser., 2005, vol. 13, pp. 330-335.

[22] Y. Ye-Lin, J. Alberola-Rubio, G. Prats-boluda, A. Perales, D. Desantes, and J. Garcia-Casado, "Feasibility and analysis of bipolar concentric recording of electrohysterogram with flexible active electrode," Ann. Biomed. Eng., vol. 43, no. 4, pp. 968-976, Apr. 2015.

[23] T. Hiyama, S. Sakurazawa, M. Toda, J. Akita, K. Kondo, and Y. Nakamura, "Motion estimation of five fingers using small concentric ring electrodes for measuring surface electromyography," in 3rd Global Conf. Consum. Electron. IEEE GCCE, 2014, pp. 376-380.

[24] M. Rafols-de-Urquia et al., "Assessment of respiratory muscle activity with surface electromyographic signals acquired by concentric ring electrodes," in Proc. 40th Annu. Int. Conf. IEEE EMBS, 2018, pp. 33503353.

[25] L. Estrada, A. Torres, J. Garcia-Casado, L. Sarlabous, G. Prats-Boluda, and R. Jane, "Time-frequency representations of the sternocleidomastoid muscle electromyographic signal recorded with concentric ring electrodes," in Proc. 38th Annu. Int. Conf. IEEE EMBS, 2016, pp. 37853788 .

[26] H. J. Hermens, B. Freriks, C. Disselhorst-Klug, and G. Rau, "Development of recommendations for SEMG sensors and sensor placement procedures," J. Electromyogr. Kinesiol., vol. 10, no. 5, pp. 361-374, Oct. 2000.

[27] L. Estrada, A. Torres, L. Sarlabous, and R. Jané, "Improvement in neural respiratory drive estimation from diaphragm electromyographic signals using fixed sample entropy," IEEE J. Biomed. Heal. Informatics, vol. 20, no. 2, pp. 476-485, Mar. 2016.

[28] D. Falla, P. Dall'Alba, A. Rainoldi, R. Merletti, and G. Jull, "Location of innervation zones of sternocleidomastoid and scalene muscles - A basis for clinical and research electromyography applications," Clin. Neurophysiol., vol. 113, no. 1, pp. 57-63, Jan. 2002.

[29] M. Ràfols-de-Urquía, L. Estrada, J. Estévez-Piorno, L. Sarlabous, R. Jané, and A. Torres, "Evaluation of a wearable device to determine cardiorespiratory parameters from surface diaphragm electromyography," IEEE J. Biomed. Heal. Informatics, vol. 23, no. 5, pp. 1964-1971, Sep. 2019.

[30] J. Pan and W. J. Tompkins, "A real-time QRS detection algorithm," IEEE Trans. Biomed. Eng., vol. 32, no. 3, pp. 230-236, Mar. 1985.

[31] L. Sarlabous et al., "Electromyography-based respiratory onset detection in COPD patients on non-invasive mechanical ventilation," Entropy, vol. 21, no. 3, p. 258, Mar. 2019.

[32] J. Lian, S. Srinivasan, H. C. Tsai, D. Wu, B. Avitall, and B. He, "Estimation of noise level and signal to noise ratio of laplacian electrocardiogram during ventricular depolarization and repolarization," Pacing Clin. Electrophysiol., vol. 25, no. 10, pp. 1474-1487, Oct. 2002.

[33] L. Estrada, A. Torres, J. Garcia-Casado, G. Prats-Boluda, and R. Jané, "Characterization of laplacian surface electromyographic signals during isometric contraction in biceps brachii," in Proc. 35th Annu. Int. Conf. IEEE EMBS, 2013, vol. 2013.

[34] K. Wang, U. Parekh, T. Pailla, H. Garudadri, V. Gilja, and T. N. Ng,
"Stretchable dry electrodes with concentric ring geometry for enhancing spatial resolution in electrophysiology," Adv. Healthc. Mater., vol. 6, no. 19, p. 1700552 , Oct. 2017.

[35] Y. Ye-Lin, J. M. Bueno-Barrachina, G. Prats-boluda, R. Rodriguez de Sanabria, and J. Garcia-Casado, "Wireless sensor node for non-invasive high precision electrocardiographic signal acquisition based on a multiring electrode," Measurement, vol. 97, pp. 195-202, Feb. 2017.

[36] C. C. Lu and P. P. Tarjan, "Pasteless, active, concentric ring sensors for directly obtained Laplacian cardiac electrograms," J. Med. Biol. Eng., vol. 22, no. 4, pp. 199-203, Dec. 2002.

[37] J. Alberola-Rubio, G. Prats-Boluda, Y. Ye-Lin, J. Valero, A. Perales, and J. Garcia-Casado, "Comparison of non-invasive electrohysterographic recording techniques for monitoring uterine dynamics," Med. Eng. Phys., vol. 35, no. 12, pp. 1736-1743, Dec. 2013.

[38] D. Farina and C. Cescon, "Concentric-ring electrode systems for noninvasive detection of single motor unit activity," IEEE Trans. Biomed. Eng., vol. 48, no. 11, pp. 1326-1334, Nov. 2001.

[39] H. Christensen, K. Søgaard, B. R. Jensen, L. Finsen, and G. Sjøgaard, "Intramuscular and surface EMG power spectrum from dynamic and static contractions," J. Electromyogr. Kinesiol., vol. 5, no. 1, pp. 27-36, Mar. 1995.

[40] A. D. de Andrade et al., "Inspiratory muscular activation during threshold therapy in elderly healthy and patients with COPD," $J$. Electromyogr. Kinesiol., vol. 15, no. 6, pp. 631-639, Dec. 2005.

[41] M. Lozano-Garcia et al., "Noninvasive assessment of inspiratory muscle neuromechanical coupling during inspiratory threshold loading," IEEE Access, vol. 7, pp. 183634-183646, Dec. 2019.

[42] M. L. Duiverman, L. a van Eykern, P. W. Vennik, G. H. Koëter, E. J. W. Maarsingh, and P. J. Wijkstra, "Reproducibility and responsiveness of a noninvasive EMG technique of the respiratory muscles in COPD patients and in healthy subjects," J. Appl. Physiol., vol. 96, no. 5, pp. 1723-1729, May 2004.

[43] J. Martin, M. Aubier, and L. A. Engel, "Effects of inspiratory loading on respiratory muscle activity during expiration," Am. Rev. Respir. Dis., vol. 125 , no. 3, pp. 352-358, Mar. 1982.

[44] A. M. Cairns and J. D. Road, "High-frequency oscillation and centroid frequency of diaphragm EMG during inspiratory loading," Respir. Physiol., vol. 112, no. 3, pp. 305-313, Jun. 1998.

[45] Y. B. Seven, C. B. Mantilla, W. Z. Zhan, and G. C. Sieck, "Nonstationarity and power spectral shifts in EMG activity reflect motor unit recruitment in rat diaphragm muscle," Respir. Physiol. Neurobiol., vol. 185, no. 2, pp. 400-409, Jan. 2013.

[46] W. G. Besio et al., "High-frequency oscillations recorded on the scalp of patients with epilepsy using tripolar concentric ring electrodes," IEEE J. Transl. Eng. Heal. Med., vol. 2, p. 2000111, Jun. 2014.

[47] A. E. Gygi and G. S. Moschytz, "Low-pass filter effect in the measurement of surface EMG," in Proc. of Comput. Based Med. Syst., 1997, pp. 183-188.

[48] M. A. Johnson, J. Polgar, D. Weightman, and D. Appleton, "Data on the distribution of fibre types in thirty-six human muscles. An autopsy study," J. Neurol. Sci., vol. 18, no. 1, pp. 111-129, Jan. 1973.

[49] M. Mizuno, "Human respiratory muscles: fibre morphology and capillary supply," Eur. Respir. J., vol. 4, no. 5, pp. 587-601, May 1991. 